\title{
Sun beams on hysterectomies
}

\author{
Liselotte Mettler • Wael Sammur • Thoralf Schollmeyer
}

Received: 5 October 2010 / Accepted: 27 December 2010 /Published online: 4 March 2011

(C) Springer-Verlag 2011

\begin{abstract}
Are hysterectomies still necessary in 2010 and why and how should they be performed? As every now and then a critical evaluation of routine surgical procedure is necessary, there it is: This review follows the "Perspectives on laparoscopic hysterectomy" by Michelle Nisolle (Gynecol Surg 7:105-107, 2010). Hysterectomies performed in the field of obstetrics and gynaecology until the nineteenth century had always a lethal end. In the twentieth century, they were perhaps too frequently performed whereas the twenty-first century has witnessed a steep decline in hysterectomy numbers. It is therefore an opportune time to review the indications for hysterectomies, hysterectomy techniques and the present and future status of this surgical procedure. There is a widespread consensus that hysterectomies are primarily to be performed in cancer cases and obstetrical chaos situations even though minimal invasive surgical technologies have made the procedure more patient-friendly than the classical abdominal opening. Today, minimally invasive hysterectomies are performed as frequently as vaginal hysterectomies, and the vaginal approach is still the first choice if the correct indications are given. It is no longer necessary to open the abdomen; this procedure has been replaced by laparoscopic surgery with multiple and single port entries. Laparoscopic and roboticassisted laparoscopic surgery can also be indicated for hysterectomies in selected patients with gynaecological
\end{abstract}

L. Mettler $(\bowtie) \cdot$ T. Schollmeyer

Department of Obstetrics and Gynaecology,

University Hospitals Schleswig-Holstein, Campus Kiel,

Kiel, Germany

e-mail: 1mettler@email.uni-kiel.de

W. Sammur

GMC, Dubai Healthcare City,

Dubai, United Arab Emirates cancers. For women of reproductive age, laparoscopic myomectomies and numerous other uterine-preserving techniques are applied in a first treatment step of menometrorrhagia, uterine adenomyosis and submucous myoma. These interventions are only followed by a hysterectomy if the pathology prevails.

Keyword Laparoscopic versus conventional hysterectomies

\section{Background history}

In contrast to the twentieth century, hysterectomy is no longer the major gynaecological surgical procedure. How has this change come about? Historical data on the first hysterectomy vary from country to country.

Probably the first documented medical opening of the abdomen took place on December 25, 1809 by Ephraim McDowell (1771-1830). Data relating to the first vaginal hysterectomy go back to the times of Soranus of Ephesus in Greece in the year $120 \mathrm{AD}$. The first successful abdominal hysterectomies in Europe were performed by Charles Clay on January 3, 1863 and Eugen Köberle on April 3, 1863 in Strasbourg. Both surgeons claimed to have performed the first successful hysterectomy, but this took some time to prove as Clay's first patient in 1843 died soon afterwards. A hysterectomy performed by Conrad Langenbeck on a mentally deficient/retarded patient could not be proved until 26 years later after a postmortem examination.

In the early twentieth century, up until 1945 , the subtotal hysterectomy as an abdominal procedure was the universal approach. This type of hysterectomy was associated with less pelvic infections, ureter lesions and other complications in the pre-antibiotic period. After these problems had been 
overcome by the development of antibiotics, total hysterectomy was introduced. The main concern was to prevent the occurrence of cervical stump cancer, even though only $0.4 \%$ of 6,600 cases were reported in the USA [2] and $0.1 \%$ in Finland [3].

Kurt Semm [4] and Tom Lyons [5] published similar data of vaginal cancer following abdominal hysterectomy, yet nobody considered removal of the vagina at hysterectomy as prophylaxis against this.

The Pfannenstiel incision introduced by Johannes Pfannenstiel from Breslau in 1900 proved to be the only real change in the abdominal procedure. This change was from the lower longitudinal abdominal incision to the lower horizontal abdominal incision. The universal acceptance of this incision occurred only after 1970 .

Laparoscopic hysterectomies are the achievement of the late twentieth century (see "Laparoscopic hysterectomy" section)

\section{Methods, techniques and findings to resect the uterus in malignant and benign indications}

Throughout the world in the US, Germany, Asia, Africa and Australia, gynaecologists have had ample opportunity over the last 40 years to become acquainted with all surgical methods of hysterectomy.

\section{Vaginal hysterectomy}

The first hysterectomy performed at the time of Soranus of Ephesus and the newest technique, performed in the twenty-first century under the name of Natural Orifice Surgery, use the vagina as the entrance and exit point. For all gynaecologists and surgeons, there are many ways to perform an operation, but the lex parsimoniae of William Ockham (1235-1350) is always valuable: "If we have different ways to solve a problem, the simplest way is the right one." Surgery is no exception.

When Langenbeck first performed a vaginal hysterectomy in 1813, the discipline of gynaecology was founded. Since then, vaginal access has been the privilege of the gynaecological surgeon.

Austria, with the brilliant schools of Schauta and Wertheim, still has the highest European rate of vaginal surgery. Shiril Sheth 2006 reports on $>5,000$ vaginal hysterectomies in his Indian experience [6]. Twenty randomized controlled trials (RCTs) comparing total abdominal hysterectomy, vaginal hysterectomy and laparoscopic hysterectomy and 16 RCTs comparing laparoscopic hysterectomies with total abdominal hysterectomies clearly stated that laparoscopic hysterectomy requires greater surgical skill. Vaginal hysterectomy leaves behind no scars and is the faster operative technique. Vaginal hysterectomy should be performed for the following indications:

1. Uterine prolapse

2. Dysfunctional uterine bleeding

3. Adenomyosis

4. Carcinoma in situ CIN3 of cervix

5. High risk with endometrial cancer

6. Cervical fibroids and uterine polyposis

\section{Operative steps}

Vaginal hysterectomy can be performed in six steps according to the situs of the patient [6].

1. Circumcision of the cervix with the scalpel after grasping the cervix with two sutures or cervical clamps. According to Joel-Cohen 1972 [7] and Stark 2006 [8], in patients with uterine prolapse, the incision of the vaginal wall can also start below the orificium urethrae externum. If the cut is deep enough, the vaginal wall can be pushed back with the finger and mobilisation is easy. If necessary, the vaginal wall can be separated from the cervix with scissors.

2. Separation of bladder from uterus and opening of the spatium vesico uterinum or the spatium recto uterinum. If the spatium vesico uterinum cannot be opened easily, it is easier to open the spatium recto uterinum with scissors until the sacrouterine ligaments are visible.

3. Clamping, dissection, suturing or coagulation of the sacrouterine ligaments. The sacrouterine ligaments and paracervical tissue must not bleed. In patients with uterine prolapse, the uterine vessels are directly visible.

4. Identification of the uterine vessels, separation by knife or scissors, suturing or, often today, the use of the biclamp, followed by sharp dissection. If the peritoneum in the area of the vesico-uterine space was not opened, it is now opened by sharp dissection.

5. Extraction of the uterus through the vagina after separation from the round ligaments, the ovarian ligaments or from the infundibulopelvic ligaments. This step is sometimes performed with clamps, dissection and suturing or with the Biclamp ${ }^{\circledR}$ (thermofusion).

6. The peritoneum is left open and only the vagina is closed with individual sutures. If necessary, a reconstruction of the pelvic floor is performed to prevent consecutive vaginal prolapse or formation of a Douglas-cele by placing an extra suture between the two sacrouterine ligaments and the vaginal stump.

According to the data collected by the Center for Disease Control in the USA $[9,10]$ the mortality rate for a vaginal hysterectomy-excluding cancer patients or obstetrical 
chaos situations-is $2.7 / 10,000$ compared to $8.6 / 10,000$ for an abdominal hysterectomy.

\section{Abdominal hysterectomy}

After the first unintended abdominal supracervical hysterectomies of Charles Clay (1843) and Ellis Burnham (1853), the first deliberate hysterectomy, with the patient surviving, was carried out in 1855 by Kimball. This was an abdominal supracervical hysterectomy. After the introduction of anaesthesia by William Morton on October 16, 1846, there were several reports of abdominal hysterectomy but with a mortality of $25 \%$. Charles Clay performed his first successful hysterectomy with a patient surviving on January 3, 1863 and Eugen Köberle on April 3, 1863. Both of these doctors are considered the fathers of abdominal hysterectomy.

In 1880 , T. G. Thomas reported on 365 collected cases of abdominal hysterectomy which revealed a staggering mortality of $70 \%$. In comparison, vaginal hysterectomy had a mortality rate of $15 \%$ in 1886. Nevertheless, in 1878, Mikulicz and Wilhelm Alexander Freund [11] provoked progress in abdominal hysterectomy. They placed three ligatures on the broad ligaments, and through the introduction of new techniques for subtotal hysterectomy, the mortality rate went down in the period 1896 until 1906, from $22 \%$ to $3.4 \%$.

In the middle of the twentieth century, apart from the change from subtotal which dominated through 1945 with Cutler and Zolenger [2] to total hysterectomy, the only change in the abdominal procedure was the almost universal adoption of the less disfiguring suprasymphysary incision introduced by Johannes Pfannenstiel.

\section{Operative steps}

The procedure of abdominal hysterectomy is tailored to the indication. The uterus has to be visualized and freed in the first step. In the second step, the round ligaments are separated from the uterus. In the third step, the adnexa are separated from the uterus or from the pelvic wall. In the fourth step, the parametrium is opened, and the bladder is pushed down in the fifth step. The uterine vessels are clamped, separated and sutured. In the sixth step, the uterus is separated from the vagina trying to preserve the uterine ligament connection. In the seventh step, the vagina is closed and the sacrouterine ligaments are fixed to/through the vagina to prevent Douglas-cele (Moskowitsch technique). In the eighth and last step, after rinsing of the minor pelvis, the visceral peritoneum is left open.

Minilaparotomy hysterectomy

The minilaparotomy procedure may be considered a timesaving technique for total hysterectomy for benign uterine pathology. It offers some of the advantages of a minimally invasive procedure (low morbidity, short hospital stay and good cosmetic results) and the benefits of open access (for example, shorter learning curve than laparoscopy). It is a minimally invasive, feasible option, particularly in countries where laparoscopic hysterectomies are not available. In many reports, minilaparotomy hysterectomy has been compared to laparotomy and laparoscopic-assisted vaginal hysterectomy $[12,13]$.

\section{Laparoscopic hysterectomy}

This technique was developed over the last 25 years. As early as 1984, our teacher, Kurt Semm, was already using laparoscopic assistance in difficult vaginal hysterectomies [14].

He called this technique laparoscopic assistance for vaginal hysterectomies. In fact, many vaginal hysterectomies were performed in Kiel with laparoscopic assistance to dissect the uterus from the round ligaments, the adnexa, the sacrouterine ligaments and the cardinal ligaments. However, worldwide discussion on laparoscopic hysterectomy began after the first published laparoscopic-assisted vaginal hysterectomy by Harry Reich in 1989 [15]. We began to perform supracervical laparoscopic hysterectomy in 1989, but the first publication did not appear until 1991 [4]. Prior to this, a few journals had turned down our submitted papers, referring to the absurdity of such a surgical technique.

Nezhat et al. in the United States described their first radical hysterectomy in 1991, but a publication did not appear until 1992 [16].

Laparoscopic hysterectomies in their different forms have been a provocation for gynaecologists for the last 20 years. Gynaecologists favour the vaginal technique, and today, the only indications remaining for abdominal operations are of a malignant nature. If the operative indication for laparoscopic hysterectomy is given and the surgeon is an experienced laparoscopist, the majority of patients can be spared a laparotomy. Figure 1 gives an overview of the combination possibilities for laparoscopic and vaginal surgery, including parailiacal and paraaortic lymphadenectomies and the subtotal hysterectomies. In comparison to conventional abdominal hysterectomy and vaginal hysterectomy, the following laparoscopic hysterectomy techniques are currently practised [6, 17-19].

1. Laparoscopic-assisted vaginal hysterectomy (LAVH)

2. Total laparoscopic hysterectomy (TLH) or laparoscopic hysterectomy (LH)

3. Intrafascial supracervical hysterectomy (CISH), subtotal or supracervical hysterectomy (LSH) 


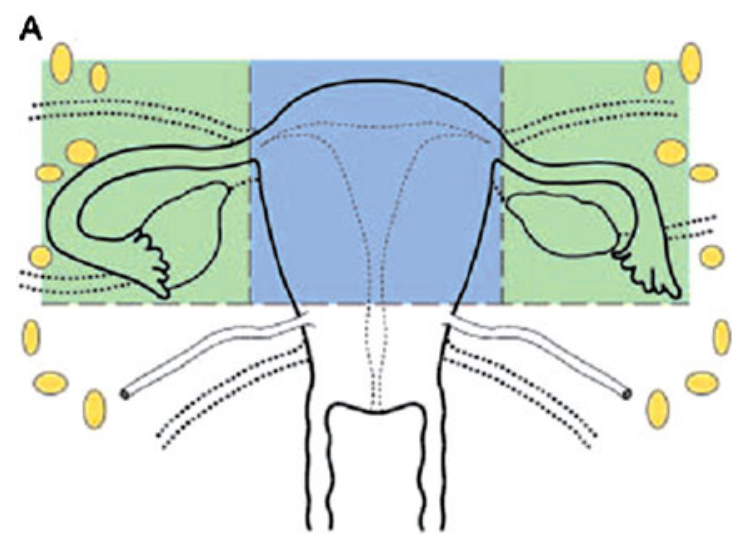

C

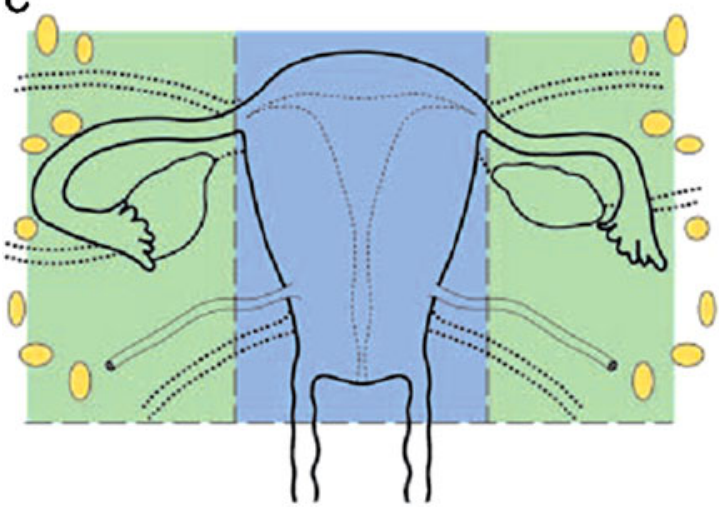

Fig. 1 Variations of laparoscopic hysterectomies (a-d). In green and blue, the laparoscopic surgery and in white, the vaginal surgery or no further surgery. a Laparoscopic resection of the uterus in the region of the round ligament, adnexa and infundibulopelvic ligament from the pelvic side wall above the cardinal ligament with or without lymphadenectomy; the uterus is extracted via the vagina. In case of laparoscopic subtotal hysterectomy (LSH), the uterus is resected above the cervix and the cervical stump remains. Morcellation of the uterus for transabdominal retraction. b Laparoscopic resection of the uterus starting from the round ligament up to the infundibulopelvic ligament. Cutting of the uterine vessels and mobilisation of the cervix up to the cervical stump with or without lymph nodes, opening of the

4. Laparoscopic radical hysterectomy (LRC) according to Wertheim or Schauta, with further specifications according to different schools

5. Robotic assistance in oncologic hysterectomy.

Robotic laparoscopic radical hysterectomy is a variation of LRH. A trachelectomy is performed in lymph node-free cases, whereby the total cervix is dissected and the vagina attached to the uterus.

\section{Laparoscopic-assisted vaginal hysterectomy}

In this case, the uterus is mobilised laparoscopically and resected transvaginally. The dissection is carried down to but excluding the uterine vessels which are secured vaginally. Similarly, uterosacral and cardinal ligaments are
B

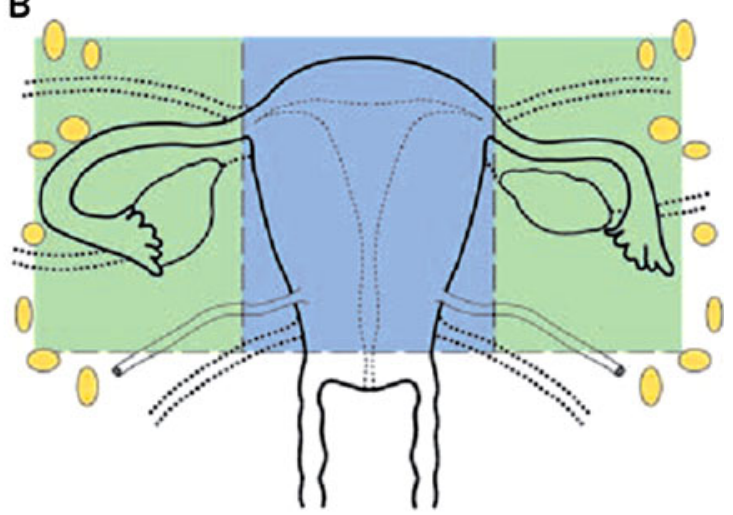

D

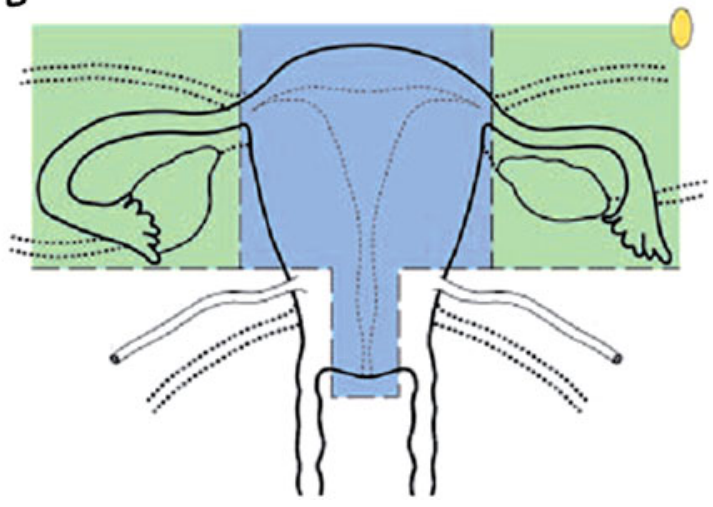

paracervical and pararectal spaces and retraction of the uterus through the vagina. c Laparoscopic resection of the uterus from the round ligament and infundibulopelvic ligament and uterine artery, visualization of paravesical and pararectal spaces with mobilisation of the uterus, including the upper third of the vagina with or without lymphadenectomy, along with the parametria and retraction of the uterus from the vagina. d Classic intrafascial subtotal hysterectomy with coring of the inner cervix and endoscopic resection of the uterus up to above the cardinal ligament. Tying only of ascending branch of the uterine artery without lymphadenectomy (for hysterectomy in benign lesions). Morcellation of the uterus for transabdominal retraction

clamped and transfixed ligated transvaginally. LAVH is performed in four laparoscopic and three vaginal surgical steps $[18,20]$.

\section{Total laparoscopic hysterectomy or LH}

Indications for TLH include benign gynaecological alterations such as fibroids, endometriosis and dysfunctional uterine bleeding in patients for whom vaginal surgery is contraindicated or cannot be performed. TLH may be performed for possible malignant indications such as early endometrial cancer, early localised small cervical cancers (trachelectomy) and also in the early stages of ovarian cancer with lymphadenectomies. The laparoscopic part consists of the preparation of the uterus and the cervix and the complete dissection of the vaginal 
stump. The individual steps of this procedure are detailed in Fig. 2.

\section{The intrafascial supracervical hysterectomy and the laparoscopic subtotal hysterectomy}

In recognition of the CISH technique, performed at the Kiel University Hospital from 1991 to 1995 [21] and still practised with interesting modifications in many countries, we would like to describe the CISH technique and the currently more frequently used LSH technique.
CISH technique Subtotal hysterectomy represents the method of choice in every form of benign uterine disease that affects only the uterus with no cervical abnormality. Annual cervical cytological surveillance is recommended to detect intracervical neoplasia which can occur if the cervix remains intact. Kurt Semm performed subtotal hysterectomy with coring of the inner cervix to totally resect the cylindrical cervical epithelium [4]. The operative steps are detailed in Fig. 3, in a sequence from A till $\mathrm{L}$. The cervix is dissected from the uterine corpus with a LINA loop [6], and the uterus is morcellated transabdominally. It is also now possible to morcellate the
Fig. 2 Total laparoscopic hysterectomy (TLH). a Multifibroid uterus, $\mathbf{b}$ dissection of the left round ligament, $\mathbf{c}$ preparation of left uterine vessels, $\mathbf{d}$ presentation of right uterine vessels, $\mathbf{e}$ ultrasound coagulation and separation of right uterine vessels, $\mathbf{f}$ ischemic uterus elevated by the uterine manipulator according to Mangeshikar, g vaginal delineation line on the ceramic cap of the Mangeshikar manipulator, $\mathbf{h}$ separation of cervix from vagina using the monopolar hook, $\mathbf{i}$ the cap becomes visible in the vaginal fornix, $\mathbf{j}$ continuous dissection of uterus from vagina, $\mathbf{k}$ extraction of a $210-\mathrm{g}$ uterus from vagina and $\mathbf{l}$ reinsertion of the manipulator tube with ceramic cap to keep the pneumoperitoneum, closure of vaginal stump with a continuous suture and two corner sutures. To prevent enterocele formation, the sacrouterine ligaments are attached to the vaginal stump
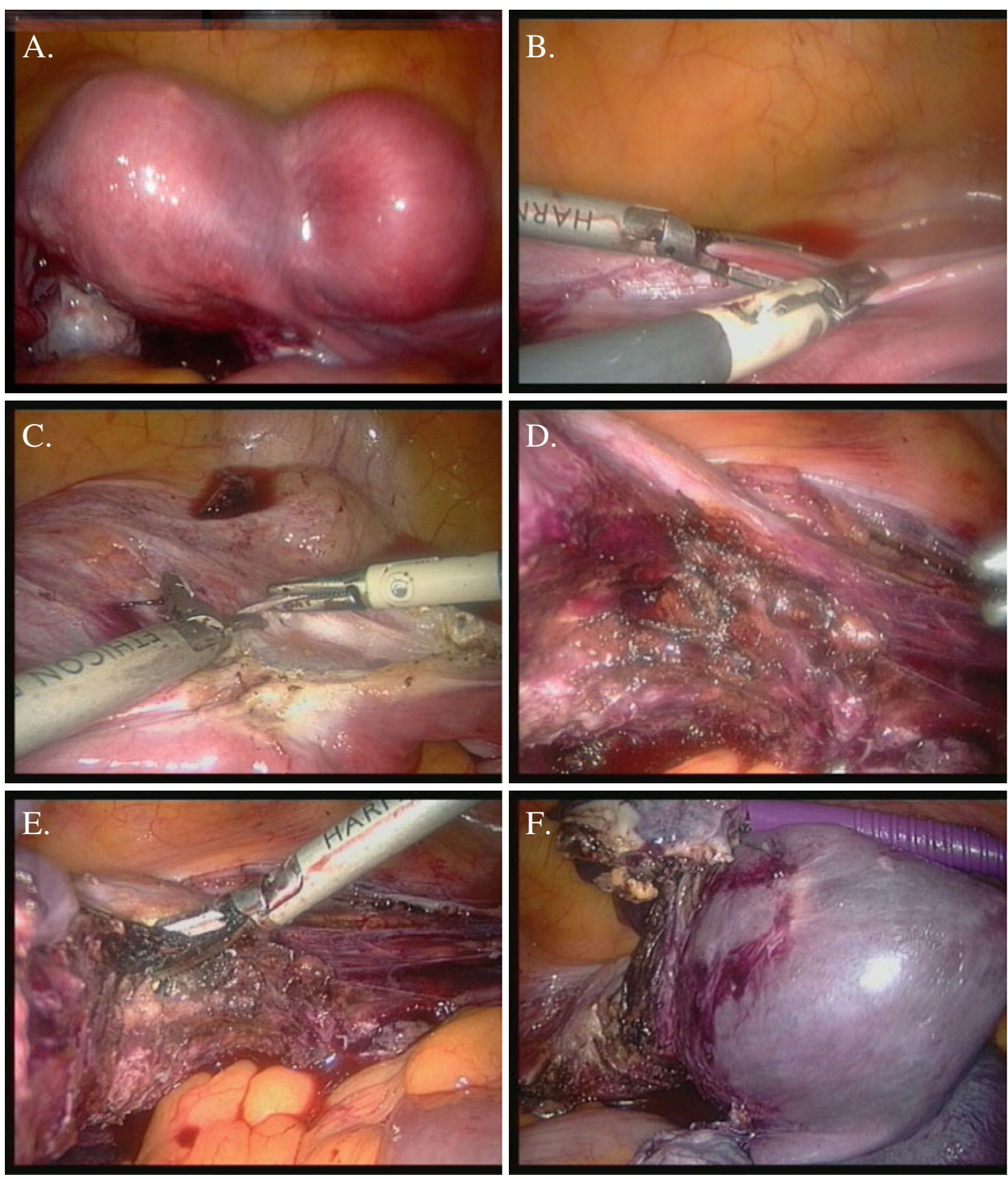

A. Multifibroid uterus

B. Dissection of the left round ligament

C. Preparation of left uterine vessels

D. Presentation of right uterine vessels

E. Ultrasound-coagulation and separation of right uterine vessels

F. Ischemic uterus elevated by the uterine manipulator according to Mangeshikar 
Fig. 2 (continued)
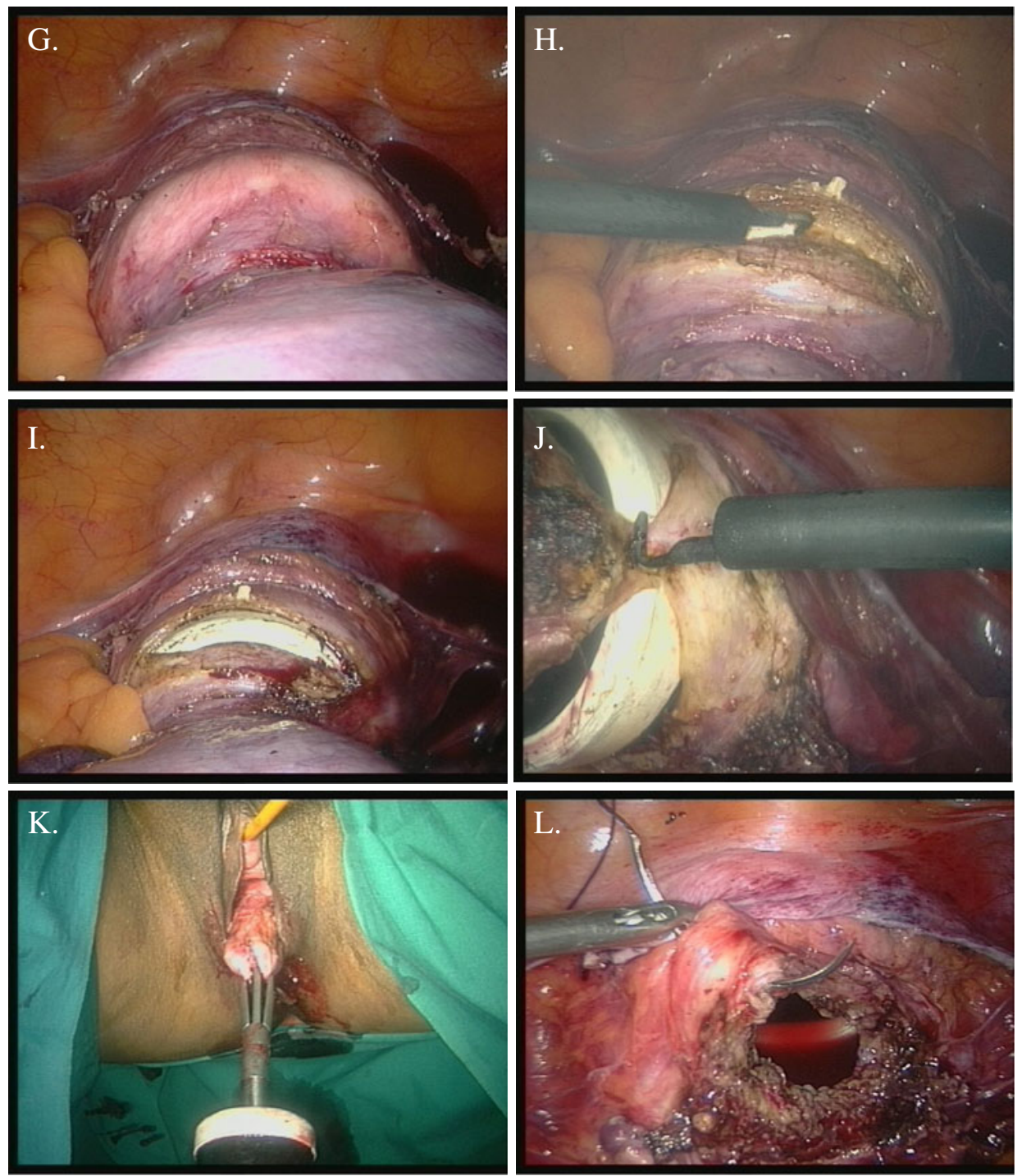

G. Vaginal delineation line on the ceramic cap of the Mangeshikar manipulator

H. Separation of cervix from vagina using the monopolar hook

I. The cap becomes visible in the vaginal fornix

J. Continuous dissection of uterus from vagina

$\mathrm{K}$. Extraction of a $210 \mathrm{~g}$ uterus from vagina

L. Reinsertion of the manipulator tube with ceramic cap to keep the pneumoperitoneum, closure of vaginal stump with a continuous suture and two corner sutures. To prevent enterocele formation, the sacrouterine ligaments are attached to the vaginal stump.

uterus transcervically with the Rotocut (Storz) after coagulation of the ascending branches of the uterine arteries, but without the positioning of Roeder loops.

LSH technique The advantage of the LSH procedure is that it can be performed on nulliparous patients, patients who have not previously had a vaginal delivery and patients who have had previous abdominal surgery. In these cases, the uterus is morcellated, but no colpotomy is performed. The technique is used mainly for fibroids, therapy-resistant dysfunctional uterine bleeding and adenomyosis. This technique is now practised routinely in Kiel according to the standardised safe minimally invasive technique [22, 23]. In a retrospective study on the clinical significance of adhesions, the effect of SprayShield as an adhesion prophylaxis has been evaluated [24]. 
Fig. 3 Classic intrafascial subtotal hysterectomy (CISH). a Multifibroid uterus, $\mathbf{b}$ dissection of left adnexa from the uterus with a stapler, $\mathbf{c}$ opening of the vesico-uterine space, $\mathbf{d}$ preparation of the bladder, e demonstration of the pericervical fascial ring, $\mathbf{f}$ transcervical and transuterine resection of a 15 $\mathrm{mm}$ tissue cylinder including the "transformation zone around an axial guide rod", g positioning of a "Roeder loop" as tourniquet to tie the ascending branches of the uterine arteries, $\mathbf{h}$ sharp dissection of cervix from the uterine corpus with scissors (or a monopolar loop as LINA Loop or Storz cervical loop), i further cervical dissection, $\mathbf{j}$ separation of uterine body from the cervix, $\mathbf{k}$ morcellation of the uterus and $\mathbf{l}$ irrigation leaving the visceral peritoneum open
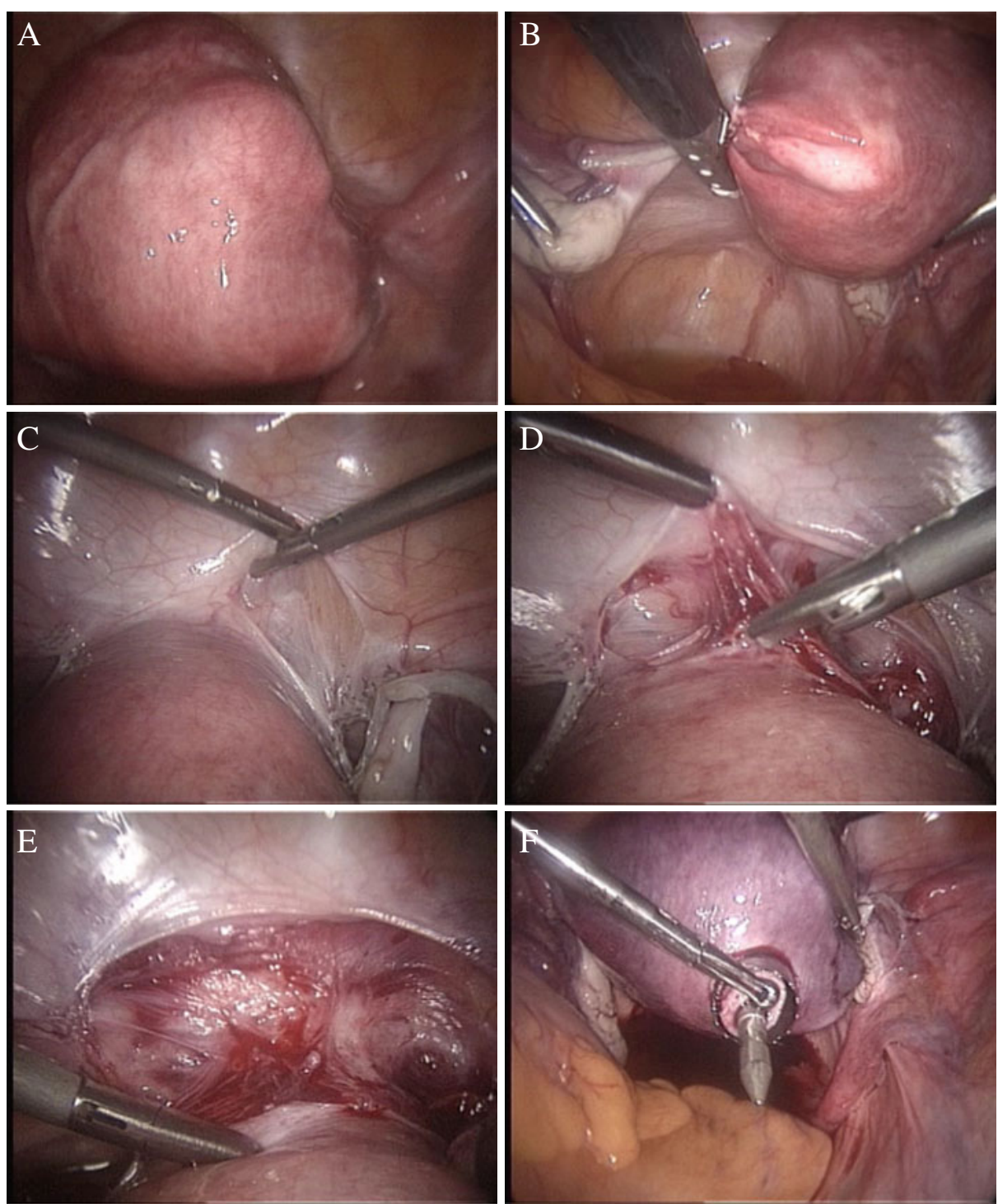

A) Multifibroid uterus

B) Dissection of left adnexa from the uterus with a stapler

C) Opening of the vesico-uterine space

D) Preparation of the bladder

E) Demonstration of the pericervical fascial ring

F) Transcervical and transuterine resection of a $15 \mathrm{~mm}$ tissue cylinder including the "transformation zone around an axial guide rod
Laparoscopic radical hysterectomy (Wertheim or Schauta technique)

Following the lead of earlier surgeons, a few skilled European and American gynaecologic surgeons have further refined the technique of radical hysterectomy, partly using robotic assistance. In addition to the Nezhat brothers [16] and Jo Childers [25, 26], who have been propagandists for radical endoscopic surgery worldwide, European colleagues such as
Daniel Dargent [27], Denny Querleu [28], Achim Schneider and Mark Possover [29] have also put intensive work into oncologic endoscopic surgery. However, it is a colleague of the third world, Shailesh Puntambekar, who has successfully brought world attention to the possibility of radical oncological surgery via laparoscopy [30].

In 1986, Dargent already began to perform laparoscopic trachelectomy in cases of small cervical cancers with no iliac lymph node metastases [27]. 
Fig. 3 (continued)
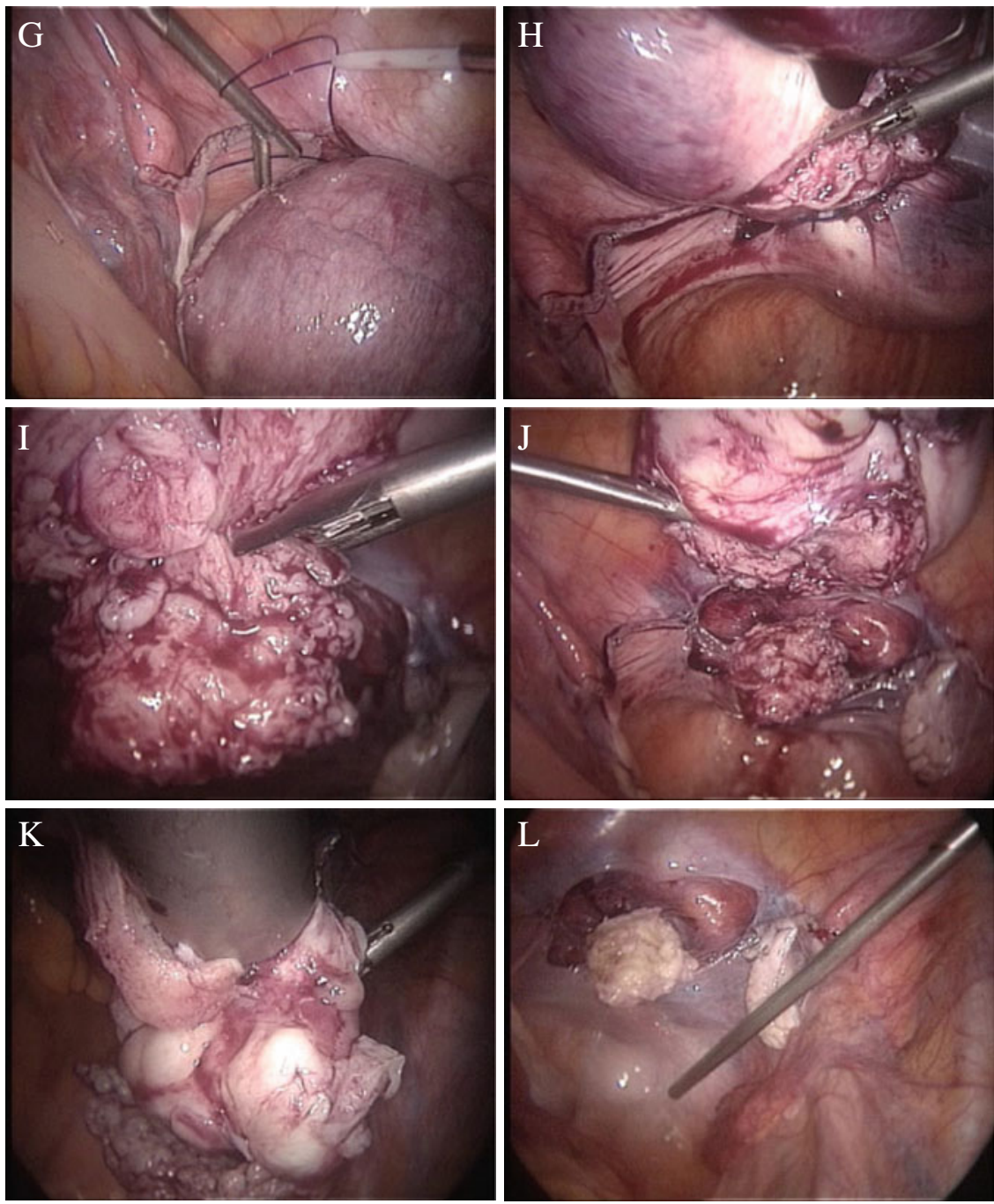

G. Positioning of a „Roeder loop“as tourniquet to tie the ascending branches of the uterine arteries

H. Sharp dissection of cervix from the uterine corpus with scissors (or a monopolar loop as LINA Loop or Storz cervical loop.

I. Further cervical dissection

J. Separation of uterine body from the cervix

K. Morcellation of the uterus

L. Irrigation leaving the visceral peritoneum open

Shailesh Putambekar performs laparoscopic radical hysterectomy not only in endometrial and early cervical cancers, but also for anterior exenteration [31, 32].

A few of us have had the opportunity to work with Shailesh Putambekar in India and in Germany. He performs excellent anterior exenterations endoscopically and proves repeatedly that radical hysterectomies are possible via the endoscopic approach.

The results of radical laparoscopic [33] and radical robotic cancer surgery [34] compare well with the outcome of radical abdominal and vaginal cancer surgery. Radical laparoscopic vaginal hysterectomy according to Schauta is successfully practised in Germany by Schneider et al. and Possover et al.
Endoscopic surgery for malignant alterations has the same chance of success as open surgery, with less surgical trauma. It depends as much on subsequent chemo or radiation therapy as open surgery does. Molecular genetic progress in the therapy of malignant disease will show the real role endoscopic surgery can take.

\section{Robotic assistance in oncologic hysterectomies}

The first robotic camera assistant used in endoscopic surgery was the automated endoscopic system for optimal positioning (AESOP; Computer Motion, Goleta, California, USA). This hand, foot or voice-controlled arm allows the 
surgeon to perform complex laparoscopic surgery faster than with an assistant holding the camera. The next surgical robot was a voice-controlled robot ZEUS (Computer Motion) that consists of AESOP to hold the camera and two additional AESOP-like units, which have been modified to hold the surgical instruments. The modern robot generation named da Vinci surgical system is based on the technologies of Computer Motion and developed by Intuitive Surgical (Mountain View, California, USA). It was approved by the US Food and Drug Administration in May 2005 for clinical use in gynaecology and was first used in reproductive gynaecology for tubal surgery [35]. There are four main components of the da Vinci surgical system [34].

(1) Surgeon's console: the surgeon sits viewing a magnified three-dimensional image of the surgical field (Fig. $4 a-c)$.

(2) Patient-side cart: this system consists of three instrument arms and one endoscope arm (Fig. 4d).

(3) Detachable instruments (EndoWrist instruments and Intuitive Masters): these detachable instruments allow
Fig. 4 a Da Vinci 2005 steering unit, $\mathbf{b}$ working position of surgeon, $\mathbf{c}$ finger movements for robotic action of instruments. Patient-side cart: $\mathbf{d}$ threedimensional rapid robotic arms ready to be connected to the three active instruments. Detachable instruments: e robotic arm with one working channel. Three-dimensional vision system: f abdominal situs at suturing
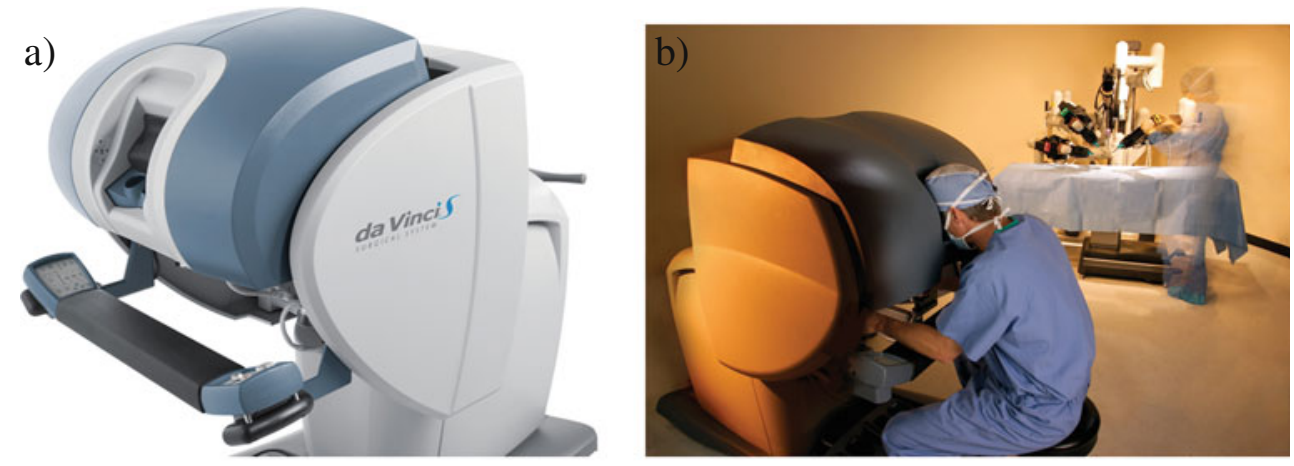

c)

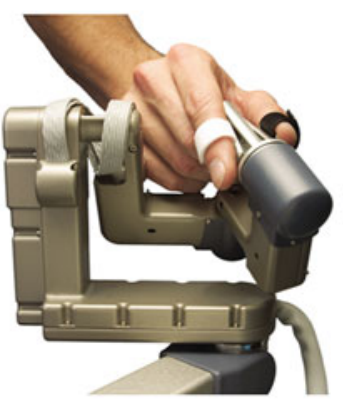

e)

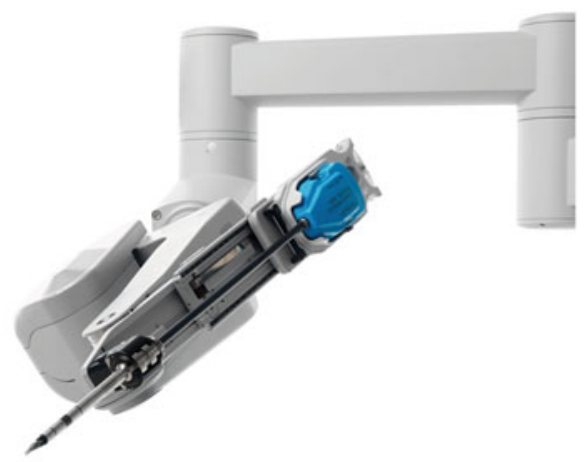

d)

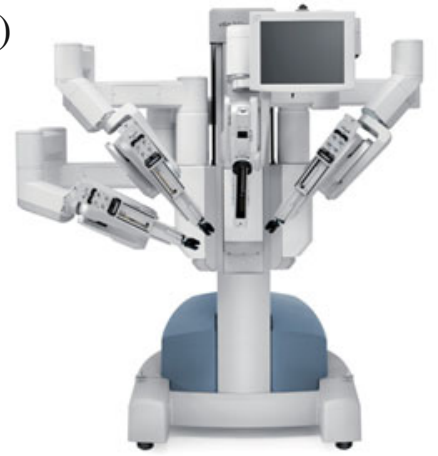

f)

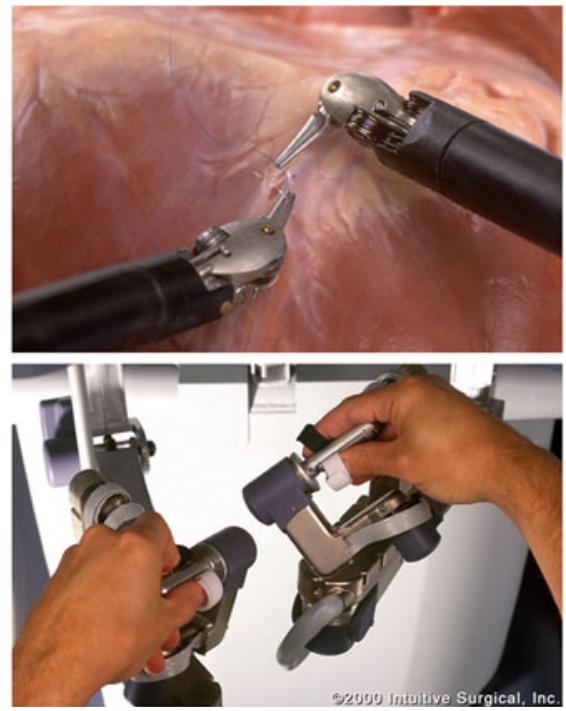


the robotic arms to manoeuvre in ways that simulate fine human movements. There are seven degrees of freedom which offer considerable choice of rotation in full circles. The surgeon is able to control the amount of force applied, which varies from a fraction of a gramme to several kilos. Tremor and scale movements are filtered out. The movements of the surgeon's hand can be translated into smaller ones by the robotic device (Fig. 4e).

(4) Three-dimensional vision system: the camera unit or endoscope arm provides enhanced three-dimensional images with the result that the surgeon knows the exact position of all instruments in relation to the anatomical structures (Fig. 4f).

The patients lie in a horizontal position with both arms tucked alongside their body. Four trocars are placed next to the optic trocar. The surgeon sits at the console and the first surgical assistant is seated in most cases on the patients' left side. This assistant controls the left accessory ports into which the instruments that are used for vessel sealing, retraction, suction, irrigation and suturing are inserted. The middle robotic arm is attached to the optical trocar with two lateral working arms to the right and one to the left. The robotic arms are connected at the beginning of the procedure and disengaged from the trocars at the end of the operation. The incisions are stitched, and the incision lines are reapproximated.

A three-dimensional vision allows the surgeon to perform ultra precise manipulations with intraabdominalarticulated instruments while providing the necessary degrees of freedom [35-42].

Results of robotic-assisted procedures in gynaecological oncology (comparative studies) The team from University of North Carolina reported on 43 robotic-assisted laparoscopic hysterectomies with pelvic and paraaortic lymph node dissection for women with endometrial cancer as a simple case report. There were no conversions to laparotomy in the robotic group compared with 3\% in the laparoscopy group. There were significantly more nodes recovered in the robotically staged patients (29.8 versus 23.2$)$. The mean blood loss in the robotic group was $63 \mathrm{ml}(25-300)$, with $45 \%$ of patients having no measurable blood loss compared with $142 \mathrm{ml}(50-700)$ in the laparoscopy group. Mean operative time was 163 min compared with 213 , and hospital stay was 1.0 compared with 1.2 days, respectively. There were $4.6 \%$ major complications in the robotic group compared with $12.8 \%$ in the laparoscopy group [43].

The team from Mayo Clinic in Scottsdale compared robotic-assisted surgery, laparoscopic surgery and open laparotomy in patients undergoing radical abdominal hysterectomy with bilateral lymphadenectomy for cervical cancer. They operated on 27 patients robotically with a mean operative time of $189.6 \mathrm{~min}, 31$ patients laparoscopically with a mean operative time of $220.4 \mathrm{~min}$ and 34 patients had an open laparotomy, with a mean operative time of $166.8 \mathrm{~min}$; the mean blood loss was 133.1, 208.4 and $443.6 \mathrm{ml}$, respectively. In this team, robotic lymphadenectomy in 27 patients resulted in a mean of 25.9 excised lymph nodes, and this appeared to be equal to that observed in laparoscopic lymphadenectomy in 31 patients, in which a mean of 25.9 lymph nodes was excised. These results compare well with open abdominal lymphadenectomy, with the mean number of excised lymph nodes being 27.7.

The robotic and laparotomy operating time was significantly shorter as compared with laparoscopy in the subgroup of patients undergoing the modified radical but not the radical technique [44]. In contrast, Boggess and colleagues reported that open abdominal pelvic lymphadenectomy in 48 patients resulted in a mean of 22.3 lymph nodes being excised, which was significantly less than the mean of 38.4 lymph nodes when robotic lymphadenectomy was used in 31 patients. As compared with laparoscopy, patients having robotically assisted surgery experienced reduced blood loss (176 versus $328 \mathrm{ml}$ ) and reduced hospitalisation (1.9 versus 2.9 days), though the lymph node count was higher for the laparoscopy group. There were no intraoperative or major postoperative complications in either group [45].

\section{Alternative techniques to hysterectomies}

Over the last 10 years, the techniques of uterine artery embolization (UAE) and magnetic resonance imaging (MRI) guidance of focussed ultrasound for uterine leiomyoma treatment have been developed. Descriptions of MRI-guided focussed ultrasound therapy treatment of fibroids indicate that it is an effective treatment for uterine leiomyomata and results in sustained symptomatic relief [46-48].

\section{Uterine artery embolization}

Is an alternative to hysterectomy in women seeking treatment for symptomatic uterine myomas $[49,50]$. It is associated with a good success rate in properly selected patients, with few major complications [51, 52].

Because of the high risk of infection, women who have had UAE and present with necrotic myoma adjacent to the endometrium should not undergo endometrial biopsy. Routine evaluation of the myoma in relation to the endometrium by means of imaging is recommended. 


\section{Endometrium ablation techniques}

Endometrium ablation techniques of the first generation= hysteroscopic endometrial resection and coagulation. In long-term studies, a success rate of $80 \%$ was achieved in reducing, but not eradicating, dysfunctional bleedings. Hysteroscopic endometrial techniques, such as the YAG laser, the resectoscope and rollerball technique (also a combination of both techniques), cryoablation and microwave techniques are available.

Second-generation methods of endometrial ablation include a number of global ablation techniques. One of the most effective appears to be the NovaSure ${ }^{\text {TM }}$ System which was introduced to Germany in 1998 by A. Gallinat [53]. It consists of a bipolar ablation device and a radiofrequency controller that enables endometrial ablation in an average of $90 \mathrm{~s}$.

\section{MRI-guided focussed ultrasound}

MRI-guided focussed ultrasound is a non-invasive treatment in which ultrasound energy, focussed on the fibroid in multiple focal spots, raises the temperature of tissue within the focal zone and causes coagulative necrosis. MRI guides and monitors the procedure, thus, providing a closed loop anatomical and thermal feedback. MRI is used to identify tumours or fibroids in the body before they are destroyed by ultrasound. Therapeutic ultrasound is a minimally invasive or non-invasive method to deposit acoustic energy into tissue.

\section{Conclusions and recommendation}

\section{Limits for hysterectomies}

Are there limits for hysterectomies or for which indications are hysterectomies still recommended? According to present medical standards, malignant disease of the ovaries, tubes and uterus is to be treated by hysterectomy. According to Dargent, trachelectomy can replace hysterectomy in younger women with early cervical cancer (smaller than $2 \mathrm{~cm}$ and without lymph node lesions in the cervix).

Contraindications for vaginal hysterectomy are a very large uterus that cannot be vaginally morcellated and nondescent of uterus. In these cases, laparoscopy, in combination with vaginal hysterectomy, can be performed. Abdominal hysterectomy has become less important and is mainly performed for cancer cases. The size of the uterus, multiple adhesions, endometriosis and obstetrical chaos situations can limit the feasibility of laparoscopic hysterectomy. In cases of massive bleeding, abdominal access is still the chosen route. Natural Orifice Surgery, with one instrument panel applied transvaginally, may open new doors for vaginal surgery.

Hysterectomy for benign indications, irrespective of surgical technique, increases the risk for stress urinary incontinence which may occur through damage to nerves, vascular supply or uterine descent [54]. It is also associated with an increased risk for subsequent pelvic organ prolapse leading to enterocele prolapse [55]. LSH or LASH seems to meet best patient satisfaction [56].

\section{Future aspects}

Considering how long Homo sapiens has inhabited the planet earth, the history of hysterectomy is a short one. This surgical technique began with a high mortality rate and a high morbidity rate, but with technological advances in the twentieth and twenty-first centuries, particularly after antisepsis and antibiotic prophylaxis eradicated infections and safe anaesthesia and infusion therapy decreased the high mortality rates, the procedure has now become very safe, with a mortality rate of approximately 12 per 10,000 [57]. Hysterectomy, with a few exceptions (cancer cases), is increasingly performed to improve quality of life, rather than to save life.

It is difficult to foresee the future, but almost certainly, other alternatives to hysterectomy will continue to evolve. For example, a better understanding of endometriosis has already produced a new therapy basis for this disease. The development of a HPV vaccine, early cervical cancer detection and the effective recognition of endometrium carcinoma also influence therapy. No surgical alternative for ovarian cancer has so far been found, and hysterectomy, in addition to lymphadenectomy and omentum resection, prevails. These techniques, however, can be performed laparoscopically [29, 58].

\section{Summary}

In the twenty-first century, abdominal hysterectomy as a surgical intervention for benign indications belongs in the past. With appropriate indications and modern morcellation techniques, even large uteri up to $1 \mathrm{~kg}$ and more can be surgically removed with laparoscopic assistance transvaginally or totally laparoscopically. Vaginal hysterectomy is still the favoured route. It should only not be used if symptoms of the patient, the expected morbidity or the inexperience of the surgeon with the vaginal technique demand laparoscopic assistance.

Malignant disease of the vagina, cervix, uterus, tubes or ovaries is the primary indication for abdominal hysterectomy as centres which are able to perform laparoscopic and 
robotic-assisted laparoscopic techniques for malignant disease are still rare. The further development of laparoscopic vaginal surgery in oncology, as developed by Dargent, remains a challenge for the endoscopic surgeon in the twenty-first century.

Alternative techniques to hysterectomy, such as endometrium ablation, have emerged and should always be considered before a hysterectomy is performed. For benign indications with an intact cervix, no endometriosis and no previous cervical surgery, laparoscopic subtotal hysterectomy leaving the cervix in place (LSH, CISH) provides a minimally invasive alternative to all other methods of total hysterectomy in benign conditions.

However, if the patient cannot have regular controls postoperatively, laparoscopic total hysterectomy is preferable as with subtotal hysterectomy regular pap or thin-prep controls are necessary.

Acknowledgement We highly acknowledge the excellent assistance of our office managers, Dawn Rüther and Nicole Guckelsberger. We thank them for their lasting and continuous support.

Declaration of interest The authors report no conflicts of interest. The authors alone are responsible for the content and writing of the paper.

\section{References}

1. Nisolle M (2010) Perspectives on laparoscopic hysterectomy. Gynecol Surg 7:105-107

2. Cutler EC, Zolenger RM (1949) Atlas of surgical operations. McMillan \& Co, New York

3. Kilkku P, Gronroos M et al (1985) Supravaginal uterine amputation with peroperative electrocoagulation of endocervical mucosa. Description of the method. Acta Obstet Gynecol Scand 64(2): 175-177

4. Semm K (1991) Hysterektomie per laparotomiam oder per pelviscopiam. Geburtshilfe Frauenheilkd 51(12):996-1003

5. Lyons TL (1993) Laparoscopic supracervical hysterectomy. A comparison of morbidity and mortality results with laparoscopically assisted vaginal hysterectomy. J Reprod Med 38(10):763-767

6. Mettler L (ed) (2007) Manual of new hysterectomy techniques. Jaypee Brothers, New Delhi

7. Joel-Cohen SJ (1972) Abdominal and vaginal hysterectomy: new techniques based on time and motion studies. Heinemann, London

8. Stark M, Gerli S et al (2006) The ten-step vaginal hysterectomy. Prog Obstet Gynaecol 17:358-368

9. Sheth SS (2002) Vaginal or abdominal hysterectomy. In: Sheth SS, Studd JW (eds) Vaginal hysterectomy. Martin Dunitz Ltd, London, pp 301-320

10. Sheth SS (2005) Vaginal hysterectomy. Best Pract Res Clin Obstet Gynaecol 19(3):307-332

11. Freund WA (1878) Bemerkungen $\mathrm{zu}$ meiner Methode der Uterusextirpation. Zbl Gynäkol 2:497-500

12. Muzii L, Basile S et al (2007) Laparoscopic-assisted vaginal hysterectomy versus minilaparotomy hysterectomy: a prospective, randomized, multicenter study. J Minim Invasive Gynecol 14 (5):610-615
13. Royo P, Alcazar JL et al (2009) The value of minilaparotomy for total hysterectomy for benign uterine disease: a comparative study with conventional Pfannenstiel and laparoscopic approaches. Int Arch Arb Med 2(1):11

14. Semm K (1984) Operationslehre für endoskopische abdominalchirurgie. Schattauer, Stuttgart

15. Reich H, DeCaprio J et al (1989) Laparoscopic hysterectomy. J Gynecol Surg 5:213-216

16. Nezhat CR, Burrell MO et al (1992) Laparoscopic radical hysterectomy with paraaortic and pelvic node dissection. Am J Obstet Gynecol 166(3):864-865

17. Mettler L, Semm K (1994) Pelviscopy and its secrets to detect and treat genital endometriosis. In: Coutinho E, Spinola P, Hanson de Moura L (eds) Progress in the management of endometriosis. Parthenon, New York, pp 327-333

18. Mettler L, Ahmed-Ebbiary N et al (2005) Laparoscopic hysterectomy: challenges and limitations. Minim Invasive Ther Allied Technol 14(3):145-159

19. Mettler L (ed) (2006) Manual for laparoscopic and hysteroscopic gynecological surgery. Jaypee Brothers, New Delhi

20. Mettler L (ed) (2002) Endoskopische Abdominalchirurgie in der Gynäkologie. Schattauer, Stuttgart

21. Semm K (1993) Hysterectomy by pelviscopy; an alternative approach without colpotomy (CASH). In: Garry R, Reich H (eds) Laparoscopic hysterectomy. Blackwell Scientific Publications, Oxford, pp 118-132

22. Salfelder A, Lueken RP et al (2005) A prospective multicenter study by the VAAO. Geburtshilfe Frauenheilkd 65:396-403

23. Bojahr B, Raatz D et al (2007) Laparoscopic supracervical hysterectomy: a standardised safe minimal invasive technique. In: Mettler L (ed) Manual of new hysterectomy techniques. Jaypee Brothers, New Delhi

24. Mettler L, Alhujeily M (2007) Role of laparoscopy in identifying the clinical significance and cause of adhesions and chronic pelvic pain: a retrospective review at the Kiel School of Gynecological Endoscopy. JSLS 11(3):303-308

25. Childers JM, Surwit EA (1992) Combined laparoscopic and vaginal surgery for the management of two cases of stage I endometrial cancer. Gynecol Oncol 45(1):46-51

26. Childers JM, Brzechffa PR et al (1993) Laparoscopically assisted surgical staging (LASS) of endometrial cancer. Gynecol Oncol 51 (1):33-38

27. Dargent D (1987) A new future for Schauta's operation through pre-surgical retroperitoneal pelviscopy. Eur J Gynaecol Oncol 8:292-296

28. Querleu D (1989) Transvaginal hysterectomy. Sub-serous technic. J Gynecol Obstet Biol Reprod 18(4):515-518

29. Kamprath S, Possover M et al (2000) Laparoscopic sentinel lymph node detection in patients with cervical cancer. Am J Obstet Gynecol 182(6):1648

30. Puntambekar SP (2007) Atlas of laparoscopic surgery in gynecologic oncology. Jaypee Brothers, New Delhi

31. Puntambekar SP, Kudchadkar RJ et al (2002) Role of pelvic exenteration in advanced and recurrent pelvic tumours. J Pelvic Surg 8(5):241-245

32. Puntambekar SP, Kudchadkar RJ (2006) Laparoscopic pelvic exenteration for advanced pelvic cancers: a review of 16 cases. Gynecol Oncol 102(3):513-516

33. Querleu D (1993) Laparoscopically assisted radical vaginal hysterectomy. Gynecol Oncol 51(2):248-254

34. Mettler L, Schollmeyer $T$ et al (2008) Robotic assistance in gynecological oncology. Curr Opin Oncol 20(5):581-589

35. Degueldre M, Vandromme J et al (2000) Robotically assisted laparoscopic microsurgical tubal reanastomosis: a feasibility study. Fertil Steril 74(5):1020-1023 
36. Sung GT, Gill IS (2001) Robotic laparoscopic surgery: a comparison of the Da Vinci and Zeus systems. Urology 58(6):893-898

37. Reynolds RK, Burke WM et al (2005) Preliminary experience with robot-assisted laparoscopic staging of gynecologic malignancies. JSLS 9(2):149-158

38. Advincula AP (2006) Surgical techniques: robot-assisted laparoscopic hysterectomy with the da Vinci surgical system. Int J Med Robot 2(4):305-311

39. Reynolds RK, Advincula AP (2006) Robot-assisted laparoscopic hysterectomy: technique and initial experience. Am J Surg 191 (4):555-560

40. Nezhat C, Saberi NS et al (2006) Robotic-assisted laparoscopy in gynecological surgery. JSLS 10(3):317-320

41. Advincula AP, Song A (2007) The role of robotic surgery in gynecology. Curr Opin Obstet Gynecol 19(4):331-336

42. Kho RM, Hilger WS et al (2007) Robotic hysterectomy: technique and initial outcomes. Am J Obstet Gynecol 197 (1):113, e1-4

43. Boggess JF, Fowler WC Jr et al (2007) Robotic assistance improves minimally invasive surgery for endometrial cancer. 38th Annual Meeting of the Society of Gynecologic Oncologists, San Diego, 3-7 Mar 2007 (Abstract no. 265)

44. Magrina JF, Kho RM et al (2008) Robotic radical hysterectomy: comparison with laparoscopy and laparotomy. Gynecol Oncol 109 (1):86-91

45. Schafer A, Boggess JF et al (2006) Type III radical hysterectomy for obese women with cervical carcinoma: robotic versus open. 37th Annual Meeting of the Society of Gynecologic Oncologists, Palm Springs, 22-26 Mar 2006 (Abstract no. 49)

46. Stewart EA, Gedroyc WM et al (2003) Focused ultrasound treatment of uterine fibroid tumors: safety and feasibility of a noninvasive thermoablative technique. Am J Obstet Gynecol 189 (1):48-54
47. Stewart EA, Rabinovici J et al (2006) Clinical outcomes of focused ultrasound surgery for the treatment of uterine fibroids. Fertil Steril 85(1):22-29

48. Stewart EA, Gostout B et al (2007) Sustained relief of leiomyoma symptoms by using focused ultrasound surgery. Obstet Gynecol 110(2 Pt 1):279-287

49. Ravina JH, Herbreteau D et al (1995) Arterial embolisation to treat uterine myomata. Lancet 346(8976):671-672

50. Ravina JH, Aymard A et al (2003) Uterine fibroids embolization: results about 454 cases. Gynécol Obstét Fertil 31(78):597-605

51. Spies JB, Spector A et al (2002) Complications after uterine artery embolization for leiomyomas. Obstet Gynecol 100(5 Pt 1):873-880

52. Mehta H, Sandhu C et al (2002) Review of readmissions due to complications from uterine fibroid embolization. Clin Radiol 57 (12):1122-1124

53. Gallinat A (2007) Endometrial ablation contra hysterectomy: who takes the decision? In: Mettler L (ed) Manual of new hysterectomy techniques. Jaypee Brothers, New Delhi, pp 1116-1120

54. Altman D, Granath F et al (2007) Hysterectomy and risk of stressurinary-incontinence surgery: nationwide cohort study. Lancet 370 (9597):1494-1499

55. Altman D, Falconer C et al (2008) Pelvic organ prolapse surgery following hysterectomy on benign indications. Am J Obstet Gynecol 198(5):572, e1-6

56. Kafy S, Al-Sannan B, Kabli N, Tulandi T (2009) Patient satisfaction after laparoscopic total or supracervical hysterectomy. Gynecol Obstet Investig 67:169-172

57. Bachmann GA (1990) Hysterectomy. A critical review. J Reprod Med 35(9):839-862

58. Querleu D, Ferron G et al (2008) Pelvic lymph node dissection via a lateral extraperitoneal approach: description of a technique. Gynecol Oncol 109(1):81-85 